\title{
Indefinite Stochastic Linear Quadratic Control and Generalized Differential Riccati Equation
}

\author{
M. Ait Rami* J. B. Moore ${ }^{\dagger}$ and Xun Yu Zhou ${ }^{\ddagger}$
}

\begin{abstract}
We consider a stochastic linear-quadratic (LQ) problem with possible indefinite cost weighting matrices for the state and the control. An outstanding open problem is to identify an appropriate Riccati-type equation whose solvability is equivalent to the solvability of this possibly indefinite LQ problem. In this paper we introduce a new type of differential Riccati equation, called the generalized (differential) Riccati equation, which in turn provides a complete solution to the indefinite LQ problem. Moreover, all the optimal feedback/open-loop controls can be identified via the solution to this Riccati equation.
\end{abstract}

Keywords. Stochastic LQ control, indefinite costs, generalized Riccati equation.

\section{Introduction}

In optimal LQ control theory, the Riccati equation approach has been used systematically to provide an optimal feedback control (see $[13,19,4]$ for the deterministic case, and $[21,5,11]$ for the stochastic case). It has been always assumed that the cost function has a positive definite weighting matrix, $R$, for the control term, and a nonnegative definite weighting matrix, $Q$, for the state term. In this case, the solvability of the Riccati

\footnotetext{
${ }^{*}$ Department of Information Engineering, The Chinese University of Hong Kong, Shatin, Hong Kong, $<$ mustapha@ie.cuhk.edu.hk>.

${ }^{\dagger}$ Research School of Information Sciences and Engineering, The Australian National University, Canberra, ACT 0200, Australia, <john.moore@syseng.anu.edu.au.>.

${ }^{\ddagger}$ Department of Systems Engineering and Engineering Management, The Chinese University of Hong Kong, Shatin, Hong Kong, <xyzhou@se.cuhk.edu.hk>. fax: 8522603-5505. Supported by the RGC Earmarked Grants CUHK 4125/97E and CUHK 4054/98E.
}

equation is both necessary and sufficient for the solvability of the underlying LQ problem. However, it was found in [7] for the first time that a stochastic LQ problem with indefinite $R$ may still be well-posed. This phenomenon has to with the deep nature of the uncertainty involved; see [7] for a detailed discussion and many examples. Some pratical applications of indefinite LQ control to portfolio selection problems and a contingent claim problem can be found in $[22,16]$ and [14], respectively. Follow-up researches on indefinite stochastic LQ control in finite time horizon have been carried out in $[8,15,9]$ to incorporate more complicated features such as random coefficients and integral constraints. Other properties concerning existence, uniqueness and asymptotic behavior of solutions to the generalized Riccati equation associated with an indefinite LQ problem are studied in [1]. The infinite-time-horizon case, where the stability becomes a crucial issue, was treated in $[2,6]$ via semidefinite programming.

In [7] it is shown that if the following type of Riccati equation, called the stochastic Riccati equation ( $t$ is suppressed),

$$
\left\{\begin{array}{l}
\dot{P}=-P A-A^{\prime} P-C^{\prime} P C \\
+\left(P B+C^{\prime} P D\right)\left(R+D^{\prime} P D\right)^{-1}\left(B^{\prime} P+D^{\prime} P C\right)-Q, \\
P(T)=H, \\
R+D^{\prime} P D>0, \quad \text { a.e. } t \in[0, T],
\end{array}\right.
$$

has a solution $P(\cdot)$, then the possibly indefinite LQ problem is well-posed and an optimal feedback control can be constructed explicitly via $P(\cdot)$. A natural question then is what we can say about the indefinite LQ problem if (1) does not have a solution at all? Note that the positive definiteness of $R+D^{\prime} P D$ in (1) is very restrictive so that it may exist an optimal solution to the LQ problem whereas (1) has no solution.

Finding an appropriate Riccati-type equation 
such that its solvability is equivalent to that of the underlying LQ problem, remains an outstanding open problem. In this paper we achieve this goal by introducing a new type of differential Riccati equation (given below) called generalized Riccati equation (GRE) where the positive definiteness constraint of $R+D^{\prime} P D$ is relaxed:

$$
\left\{\begin{array}{l}
\dot{P}=-P A-A^{\prime} P-C^{\prime} P C \\
+\left(P B+C^{\prime} P D\right)\left(R+D^{\prime} P D\right)^{\dagger}\left(B^{\prime} P+D^{\prime} P C\right)-Q, \\
P(T)=H, \\
\left(\left(R+D^{\prime} P D\right)\left(R+D^{\prime} P D\right)^{\dagger}-I\right)\left(B^{\prime} P+D^{\prime} P C\right)=0, \\
R+D^{\prime} P D \geq 0, \text { a.e. } t \in[0, T],
\end{array}\right.
$$

where the sign $\dagger$ represents the Moore-Penrose pseudo-inverse [18]. This new Riccati equation turns out to be the right one for studying indefinite LQ problems and permits us to obtain all possible optimal controls.

It is worth mentioning that even for deterministic singular LQ problems (see $[20,12,17,10]$ among others), which is a special case of the problem treated in this paper, our formulation and results are still new.

The remainder of this paper is organized as follows. Section 2 formulates the indefinite stochastic LQ problem and gives some preliminaries. Section 3 shows that the solvability of the GRE is sufficient for the well-posedness of the LQ problem and the existence of an optimal control. Moreover, all the optimal controls are identified via the solution of the GRE. Sections 4 shows that the solvability of the GRE is also necessary for the existence of optimal linear feedback controls as well as optimal open-loop controls. Section 5 provides an illustrative example. Finally, Section 6 gives some concluding remarks.

\section{Problem Formulation}

Let $\left(\Omega, \mathcal{F}, \mathcal{P} ; \mathcal{F}_{t}\right)$ be a given filtered probability space with a standard Brownian motion $W(t)$ on $[0, T]$ (with $W(0)=0$ ). In order to simplify exposition we assume that the Brownian motion is one-dimensional. There is no essential difficulty with the multi-dimensional case. $L_{\mathcal{F}}^{2}\left(0, T ; \mathbf{R}^{p}\right)$ is the set of $\mathcal{F}_{t^{-}}$adapted $\mathbf{R}^{p}$-valued Lebesgue measurable process $\phi(\cdot)$ on $[0, T]$, such that $E \int_{0}^{T} \phi(t, \omega)^{\prime} \phi(t, \omega) d t<+\infty$.
Consider the following linear Ito stochastic differential equation

$$
\left\{\begin{aligned}
d x(t) & =[A(t) x(t)+B(t) u(t)] d t \\
& +[C(t) x(t)+D(t) u(t)] d W(t), \\
x(s)= & y
\end{aligned}\right.
$$

where $(s, y) \in[0, T) \times \mathbf{R}^{n}$ are the initial time and initial state, respectively, and $u(\cdot)$, the admissible control, is any element in $U_{a d} \equiv$ $L_{\mathcal{F}}^{2}\left(0, T ; \mathbf{R}^{n_{u}}\right)$. The matrices $A, B, C, D$ are assumed to be bounded.

For each $(s, y)$ and $u(\cdot) \in U_{a d}$, the associated cost is

$$
\begin{aligned}
& J(s, y ; u(\cdot))=E\left[x(T)^{\prime} H x(T)\right] \\
& \quad+E\left\{\int_{s}^{T}\left[x(t)^{\prime} Q(t) x(t)+u(t)^{\prime} R(t) u(t)\right] d t\right\} .
\end{aligned}
$$

The matrices $Q, R$ and $H$ are assumed to be bounded. Our objective is to minimize the cost function $J(s, y ; u(\cdot))$, for a given $(s, y) \in[0, T) \times$ $\mathbf{R}^{n}$, over all $u(\cdot) \in U_{a d}$. The value function is defined as

$$
V(s, y)=\inf _{u(\cdot) \in U_{a d}} J(s, y ; u(\cdot)) .
$$

Definition 2.1 The optimization problem (3)(5) is called well-posed if

$$
V(s, y)>-\infty, \quad \forall(s, y) \in[0, T) \times \mathbf{R}^{n} .
$$

An admissible pair $\left(x^{*}(\cdot), u^{*}(\cdot)\right)$ is called optimal (with respect to the initial condition $(s, y)$ ) if $u^{*}(\cdot)$ achieves the infimum of $J(s, y ; u(\cdot))$.

Using Itô's formula, we have

Lemma 2.1 Let $M(\cdot)$ be a given continuously differentiable (in $t)$ matrix function taking values in $\mathcal{S}^{n}$. Then for any admissible pair $(x(\cdot), u(\cdot))$ of the system (3), we have

$$
\begin{aligned}
& E\left[x(t)^{\prime} M x(T)\right]-y^{\prime} M(s) y= \\
& E \int_{s}^{T}\left[x^{\prime}\left(\dot{M}+A^{\prime} M+M A+C^{\prime} M C\right) x\right](t) d t \\
& +E \int_{s}^{T}\left[2 u^{\prime}\left(B^{\prime} M+D^{\prime} M C\right) x+u^{\prime} D^{\prime} M D u\right](t) d t .
\end{aligned}
$$

The following will be used in the sequel. 
Lemma 2.2 (Extended Schur's lemma [3] ) Let matrices $M=M^{\prime}, N$ and $R=R^{\prime}$ be given with appropriate sizes. Then the following conditions are equivalent:

(i) $M-N R^{\dagger} N^{\prime} \geq 0, R \geq 0, N\left(I-R R^{\dagger}\right)=0$.

(ii) $\left[\begin{array}{ll}M & N \\ N^{\prime} & R\end{array}\right] \geq 0$.

Lemma 2.3 Let matrices $L, M$ and $N$ be given with appropriate sizes. Then the following matrix equation

$$
L X M=N
$$

has a solution $X$ if and only if

$$
L L^{\dagger} N M^{\dagger} M=N .
$$

Moreover, any solution to (7) is represented by

$$
X=L^{\dagger} N M^{\dagger}+S-L^{\dagger} L S M M^{\dagger},
$$

where $S$ is a matrix with an appropriate size.

\section{Sufficiency of GRE}

We show that the solvability of the GRE (2) is sufficient for the well-posedness of the LQ problem as well as for the existence of optimal controls.

Theorem 3.1 If the GRE (2) admits a solution $P(\cdot)$, then the stochastic $L Q$ problem (3)-(5) is well-posed. Moreover, the set of all the optimal controls with respect to the initial $(s, y) \in[0, T) \times$ $\mathbf{R}^{n}$ is determined by the following (parameterized by $(Y, z))$ :

$$
\begin{aligned}
u_{(Y, z)}= & -\left\{\left[R+D^{\prime} P D\right]^{\dagger}\left[B^{\prime} P+D^{\prime} P C\right]+Y\right. \\
& \left.-\left[R+D^{\prime} P D\right]^{\dagger}\left[R+D^{\prime} P D\right] Y\right\} x \\
& +z-\left[R+D^{\prime} P D\right]^{\dagger}\left[R+D^{\prime} P D\right] z
\end{aligned}
$$

where $Y \in L_{\mathcal{F}}^{2}\left(s, T ; \mathbf{R}^{n_{u} \times n}\right)$ and $z \in$ $L_{\mathcal{F}}^{2}\left(s, T ; \mathbf{R}^{n_{u}}\right)$. Furthermore, the value function is uniquely determined by $P(\cdot)$ :

$$
V(s, y) \equiv \inf _{u(\cdot) \in U_{a d}} J(s, y ; u(\cdot))=y^{\prime} P(s) y .
$$

Proof : Let $P(\cdot)$ be a solution of the GRE (2). Applying Lemma 2.1, then we have

$$
\begin{aligned}
& J(s, y ; u(\cdot))=y^{\prime} P(s) y \\
& +E \int_{s}^{T}\left[x^{\prime}\left(\dot{P}+P A+A^{\prime} P+C^{\prime} P C+Q\right) x\right. \\
& \left.+2 u^{\prime}\left(B^{\prime} P+D^{\prime} P C\right) x+u^{\prime}\left(D^{\prime} P D+R\right) u\right](t) d t .
\end{aligned}
$$

Now, let $Y(\cdot) \in L_{\mathcal{F}}^{2}\left(s, T ; \mathbf{R}^{n_{u} \times n}\right)$ and $z(\cdot) \in$ $L_{\mathcal{F}}^{2}\left(s, T ; \mathbf{R}^{n_{u}}\right)$ be given. Define

$$
\begin{gathered}
L_{1}=Y-\left(R+D^{\prime} P D\right)^{\dagger}\left(R+D^{\prime} P D\right) Y, \\
L_{2}=z-\left(R+D^{\prime} P D\right)^{\dagger}\left(R+D^{\prime} P D\right) z .
\end{gathered}
$$

Then we obtain

$$
\begin{aligned}
& \left(R+D^{\prime} P D\right) L_{i}=\left(R+D^{\prime} P D\right)^{\dagger} L_{i}=0, \\
& \left(P B+C^{\prime} P D\right) L_{i}=0, i=1,2 .
\end{aligned}
$$

So that (12) leads to

$$
\begin{aligned}
& J(s, y ; u(\cdot))=y^{\prime} P(s) y \\
& +E \int_{s}^{T}\left\{\left[u+\left(R+D^{\prime} P D\right)^{\dagger}\left(B^{\prime} P+D^{\prime} P C\right) x\right.\right. \\
& \left.+L_{1} x+L_{2}\right]^{\prime}\left(R+D^{\prime} P D\right)\left[u+\left(R+D^{\prime} P D\right)^{\dagger}\right. \\
& \left.\left.\quad\left(B^{\prime} P+D^{\prime} P C\right) x+L_{1} x+L_{2}\right]\right\}(t) d t .
\end{aligned}
$$

Hence, $J(s, y ; u(\cdot))$ is minimized by the control given by (10) with the optimal index value $y^{\prime} P(s) y$. Now we show is that any optimal control can be represented by (10) for some $Y(\cdot)$ and $z(\cdot)$. Let $u(\cdot)$ be an optimal control, then we can see that the integrand in the right hand side of (14) must be zero almost everywhere in $t$. This implies

$$
\begin{aligned}
\left(R+D^{\prime} P D\right) u= & \left(R+D^{\prime} P D\right)^{\dagger}\left(B^{\prime} P+D^{\prime} P C\right) x \\
& +L_{1} x+L_{2},
\end{aligned}
$$

Applying Lemma 2.3 to solve the above equation in $u(t)$, we obtain (10).

Corollary 3.1 The optimal controls are obtained in the following special cases:

(i) If $R(t)+D(t)^{\prime} P(t) D(t) \equiv 0$, a.e. $t \in[s, T]$, then any admissible control is optimal.

(ii) If $R(t)+D(t)^{\prime} P(t) D(t)>0$, a.e. $t \in[s, T]$, then there is a unique optimal control that is given by the following linear feedback law:

$$
u=-\left(R+D^{\prime} P D\right)^{-1}\left(B^{\prime} P+D^{\prime} P C\right) x .
$$


An immediate consequence of Theorem 3.1 is the uniqueness of the solution to the generalized Riccati equation (2).

Corollary 3.2 If there is a solution to the generalized Riccati equation (2), then it must be the only solution to (2).

Proof : Let $P_{1}(\cdot)$ and $P_{2}(\cdot)$ be two solutions of the generalized Riccati equation (2). Then Theorem 3.1 implies that

$$
y^{\prime} P_{1}(s) y=y^{\prime} P_{2}(s) y, \quad \forall y \in \mathbf{R}^{n}, \quad \forall s \in[0, T] .
$$

Hence $P_{1}(t) \equiv P_{2}(t)$.

\section{Necessity of GRE}

First we need the following lemma.

Lemma 4.1 Assume that the LQ problem (3)(5) is well-posed. Then there exists a symmetric matrix function $P(\cdot)$ such that

$$
V(s, y)=y^{\prime} P(s) y, \quad \forall(s, y) \in[0, T] \times \mathbf{R}^{n} .
$$

Moreover, assume that $Q(t)$ and $R(t)$ are continuous in $t$, and for any initial $(s, y) \in[0, T] \times \mathbf{R}^{n}$ the LQ problem (3)-(5) has an optimal open-loop control that is continuous in $t$, then the matrix function $P(\cdot)$ satisfying (16) is differentiable on $[0, T]$.

Proof : First, (16) can be shown by a simple adaptation of the well-known result in the deterministic case (see, e.g., $[10,4]$ ). Moreover, since the value function $V(s, y)$ is continuous in $s$, so is $P(\cdot)$. Next, fix $(s, y)$ and let $\left(u_{*}(\cdot), x_{*}(\cdot)\right)$ be an optimal solution of (3)-(5) with respect to the initial condition $x(s)=y$ with $u_{*}(\cdot)$ continuous. Then the dynamic programming optimality principle yields

$$
\begin{aligned}
& V(s, y)=E \int_{s}^{s+h}\left[x_{*}^{\prime} Q x_{*}+u_{*}^{\prime} R u_{*}\right](t) d t \\
& +E\left[V\left(s+h, x_{*}(s+h)\right)\right], \quad \forall h \geq 0 .
\end{aligned}
$$

Making use of (16)-(17), we have

$$
\begin{aligned}
& \frac{1}{h} E\left[y^{\prime} P(s+h) y-y^{\prime} P(s) y\right]= \\
& -\frac{1}{h} E\left[y^{\prime} P(s+h) y-x_{*}(s+h)^{\prime} P(s+h) y+\ldots\right. \\
& x_{*}(s+h)^{\prime} P(s+h) y-x_{*}(s+h)^{\prime} P(s+h) x_{*}(s+h) \\
& -\int_{s}^{s+h}\left[x_{*}(t)^{\prime} Q(t) x_{*}(t)+u_{*}(t)^{\prime} R(t) u_{*}(t)\right] d t .
\end{aligned}
$$

Noting that $P(\cdot)$ and $x_{*}(\cdot)$ are continuous, and the integrand above is continuous in $t$ by the assumptions, we can show by a standard argument that the limit of each of the three terms on the righthand side of the above equation exists as $h$ goes to zero. Therefore $\lim _{h \rightarrow 0} \frac{1}{h}\left[y^{\prime} P(s+h) y-y^{\prime} P(s) y\right]$ exists. Since $y$ is arbitrary, $P(s)$ is differentiable at $s \in[0, T]$.

The assumption that the optimal control is continuous in $t$ is a rather technical one. From the above proof we can see that only the continuity of the control at the initial time $s$ is actually needed. On the other hand, if we assume that $B(t), C(t), D(t)$ and $R(t)$ are continuous, then by (10) the existence of a continuous optimal openloop control is really necessary for the solvability of the GRE (2).

The following is the main result of this section.

Theorem 4.1 Assume that $B(t), C(t), D(t), Q(t)$ and $R(t)$ are continuous in $t$. Then the $L Q$ problem (3)-(5) has a continuous optimal open-loop control for any initial $(s, y) \in[0, T] \times \mathbf{R}^{n}$ if and only if the GRE (2) has a solution $P(\cdot)$. Moreover, any optimal control is given by (10).

Proof : The sufficiency part follows from Theorem 3.1. Since the LQ problem is well-posed, Lemma 4.1 yields that there exists a symmetric matrix function $P(\cdot)$ such that

$$
V(s, y)=y^{\prime} P(s) y, \quad \forall(s, y) \in[0, T) \times \mathbf{R}^{n} .
$$

Moreover, by the assumption and Lemma 4.1, $P(\cdot)$ is differentiable. Using the dynamic programming principle we have for all $u(\cdot) \in U_{a d}$

$$
\begin{aligned}
V(s, y) \leq & \left.E \int_{s}^{s+h} x(t)^{\prime} Q(t) x(t)+u(t)^{\prime} R(t) u(t)\right] d t \\
& +E[V(s+h, x(s+h))], \quad \forall h \geq 0 .
\end{aligned}
$$

Applying Ito's formula to $V(t, x(t))$, using the above inequality, and employing Lemma 2.1, we obtain

$$
E \int_{s}^{s+h}\left(\begin{array}{l}
x \\
u
\end{array}\right)^{\prime} M(P)\left(\begin{array}{l}
x \\
u
\end{array}\right)(t) d t \geq 0,
$$

where $M(P)$ represents the matrix

$$
\left[\begin{array}{c|c}
\dot{P}+A^{\prime} P+P A+C^{\prime} P C+Q & P B+C^{\prime} P D \\
\hline B^{\prime} P+D^{\prime} P C & R+D^{\prime} P D
\end{array}\right] .
$$


Taking $u(t) \equiv \bar{u} \in \mathbf{R}^{n_{u}}$, and then dividing both sides by $h$ and letting $h \rightarrow 0$, we obtain for a.e. $s \in[0, T]$

$$
\left(\begin{array}{l}
y \\
\bar{u}
\end{array}\right)^{\prime} M(P)(s)\left(\begin{array}{l}
y \\
\bar{u}
\end{array}\right) \geq 0 .
$$

Since $y \in \mathbf{R}^{n}$ and $\bar{u} \in \mathbf{R}^{n_{u}}$ are arbitrary, we obtain for a.e. $t \in[0, T]$

$$
\left[\begin{array}{c|c}
\dot{P}+A^{\prime} P+P A+C^{\prime} P C+Q & P B+C^{\prime} P D \\
\hline B^{\prime} P+D^{\prime} P C & R+D^{\prime} P D
\end{array}\right] \geq 0 .
$$

Applying Lemma 2.2 to (18), we have

$$
\begin{aligned}
& \dot{P}+P A+A^{\prime} P+C^{\prime} P C \\
& -\left(P B+C^{\prime} P D\right)\left(R+D^{\prime} P D\right)^{\dagger}\left(B^{\prime} P+D^{\prime} P C\right)+Q \geq 0, \\
& \left(\left(R+D^{\prime} P D\right)\left(R+D^{\prime} P D\right)^{\dagger}-I\right)\left(B^{\prime} P-D^{\prime} P C\right)=0, \\
& R+D^{\prime} P D \geq 0, \text { a.e. } t \in[0, T] .
\end{aligned}
$$

Now, Let $\left(x_{*}(\cdot), u_{*}(\cdot)\right)$ be an optimal open-loop control for (3)-(5) with respect to the initial condition $x(s)=y$. Applying Lemma 2.1 to $P(\cdot)$, we have

$$
\begin{aligned}
& V(s, y)=y^{\prime} P(s) y \\
& +E \int_{s}^{T}\left[x _ { * } ^ { \prime } \left(\dot{P}+P A+A^{\prime} P+C^{\prime} P C+Q\right.\right. \\
& \left.\left.-\left(P B+C^{\prime} P D\right)\left(R+D^{\prime} P D\right)^{\dagger}\left(B^{\prime} P+D^{\prime} P C\right)\right) x_{*}\right](t) d t \\
& +E \int_{s}^{T}\left[u_{*}+\left(R+D^{\prime} P D\right)^{\dagger}\left(B^{\prime} P+D^{\prime} P C\right) x_{*}\right]^{\prime} \\
& \left(R+D^{\prime} P D\right)\left[u_{*}+\left(R+D^{\prime} P D\right)^{\dagger}\left(B^{\prime} P+D^{\prime} P C\right) x_{*}\right](t) d t .
\end{aligned}
$$

By virtue of the relation $V(s, y)=y^{\prime} P(s) y$ and (19)-(20), we obtain

$$
\begin{aligned}
& \dot{P}=-P A+A^{\prime} P-C^{\prime} P C \\
& +\left(P B+C^{\prime} P D\right)\left(R+D^{\prime} P D\right)^{\dagger}\left(B^{\prime} P+D^{\prime} P C\right)-Q .
\end{aligned}
$$

This completes the proof.

\section{An Example}

Consider the following one-dimensional LQ problem

$$
\begin{aligned}
& \min J=E\left\{\int_{0}^{1}\left[q(t) x(t)^{2}+r(t) u(t)^{2}\right] d t+h x(1)^{2}\right\} \\
& \text { subject to } \\
& \left\{\begin{array}{c}
d x(t)=[a(t) x(t)+b(t) u(t)] d t \\
+[c(t) x(t)+d(t) u(t)] d W(t), \\
x(0)=x_{0} \in b f R,
\end{array}\right.
\end{aligned}
$$

where the coefficients are such that $d(t) \neq 0, \forall t \in$ $[0,1], b(t)+d(t) c(t) \equiv 0, q(t)<0$ and $2 a(t)+$ $c(t)^{2}+q(t)>0, \quad \forall t \in[0,1]$. Take $r(t)=$ $-d(t)^{2} p(t)$, with

$p(t)=\frac{e^{\left[2 a(t)+c(t)^{2}\right](1-t)}\left[2 h a(t)+h c(t)^{2}+q(t)\right]-q(t)}{2 a(t)+c(t)^{2}}$

is the solution to the following equation

$$
\dot{p}(t)+\left[2 a(t)+c(t)^{2}\right] p(t)+q(t)=0, \quad p(1)=h .
$$

It is easy to verify directly that (23) is exactly the generalized Riccati equation in the present case (note that the singularity arises because $\left.r(t)+d(t)^{2} p(t) \equiv 0\right)$. Therefore by Theorem 3.1 and Corollary 3.1-(i), the LQ problem is wellposed, and any admissible control is optimal with an optimal cost $p(s) y^{2}$.

Furthermore, since $2 a(t)+c(t)^{2}>0$, we have from (22) that $p(t) \geq h, \forall t \in[0,1]$. Hence, if $h$ is chosen such that $h<0$ then $p(t)$ could be negative. On the other hand, if $h>0$, then $p(t)>0$ and $r(t)=-d(t)^{2} p(t)<0$. In this case, both $q(t)$ and $r(t)$ are negative but the LQ problem is well-posed. The essential reason behind this phenomenon is that the positive terminal cost $h x(1)^{2}$ outweighs the negative running cost.

\section{Conclusion}

Standard LQ theory, which has proved so useful for control applications in the last decades, has been extended here to signal models with multiplicative noises in both state and control, and with quadratic weights that are fundamentally different from those in the literature. Such models better approximate nonlinear stochastic systems and arise naturally in areas of current interest such as in finance. A new Riccati equation is introduced in this paper as an appropriate vehicle for identifying optimal controls and calculating the optimal cost value.

\section{References}

[1] M. Ait Rami, X. Chen, J.B. Moore, and X.Y. Zhou. Solvability and asymptotic behavior of 
generalized Riccati equations arising in indefinite stochastic LQ controls. IEEE Trans. Autom. Contr., Vol. AC-46 (2001), pp. 428440.

[2] M. Ait Rami and X. Y. Zhou. Linear matrix inequalities, Riccati equations, and indefinite stochastic linear quadratic control. IEEE Trans. Aut. Contr., 45:1131-1143, 2000.

[3] A. Albert. Conditions for positive and nonnegative definiteness in terms of pseudoinverse. SIAM J. Appl. Math., 17:434-440, 1969.

[4] B.D.O. Anderson and J.B. Moore. Optimal Control: Linear Quadratic Methods. Prentice-Hall, Englewood Cliffs, 1989.

[5] M. Athens. Special issues on linearquadratic-Gaussian problem. IEEE Trans. Auto. Contr., AC-16:527-869, 1971.

[6] M. Ait Rami, X. Y. Zhou and J. B. Moore. Well-posedness and Attainability of Indefinite Stochastic Linear Quadratic Control in Infinite Time Horizon. Syst. \& contr. letters. 41:123-133, 2000.

[7] S. Chen, X. Li and X. Y. Zhou. Stochastic linear quadratic regulators with indefinite control weight costs. SIAM J. Contr. Optim., 36:1685-1702, 1998.

[8] S. Chen and X. Y. Zhou. Stochastic linear quadratic regulators with indefinite control weight costs. II. SIAM J. Contr. Optim., to appear.

[9] S. Chen and J. Yong. General stochastic linear quadratic control problems. Preprint.

[10] D. Clements, B.D.O. Anderson and P. J. Moylan. Matrix inequality solution to linear-quadratic singular control problems. IEEE Trans. Auto. Contr., 55-57, 1977.

[11] M.H.A. Davis. Linear Estimation and Stochastic Control. Chapman and Hall, London, 1977.
[12] D. H. Jacobson. Totally singular quadratic minimization problems. IEEE Trans. Auto. Contr., 16:651-658, 1971.

[13] R. E. Kalman. Contribution to the theory of optimal control. Bol. Soc. Mat. Mex., 5:102$119,1960$.

[14] M. Kohlmann and X. Y. Zhou. Relationship between backward stochastic differential equations and stochastic controls: An LQ approach. SIAM J. Contr. Optim., to appear.

[15] A.E.B. Lim and X.Y. Zhou. Stochastic optimal LQR control with integral quadratic constraints and indefinite control weights. IEEE Trans. Automat. Contr., 44:359-369, 1999.

[16] A.E.B. Lim and X.Y. Zhou. Mean-variance portfolio selection with random parameters. Preprint.

[17] B. P. Molinari. Nonnegativity of quadratic functional. SIAM J. Contr., 13:792-806, 1975 .

[18] R. Penrose. A generalized inverse of matrices. Proc. Cambridge Philos. Soc., 52:17-19, 1955.

[19] V. M. Popov. Hyperstability and optimality of automatic systems with several control functions. Rev. Roum. Sci. Tech., Ser. Electrotech. Energ., 9:629-690, 1964.

[20] J. C. Willems. Least squares stationary control and the algebraic Riccati equation. IEEE Trans. Aut. Control, 16:621-234, 1971.

[21] W. M. Wonham. On a matrix Riccati equation of stochastic control. SIAM J. Contr., 6:312-326, 1968.

[22] X. Y. Zhou and D. Li. Explicit efficient frontier of a continuous-time mean-variance portfolio selection problem. Control of Distributed Parameter and Stochastic Systems, S. Chen et al. (Eds.), Kluwer, 323-330, 1999. 